\title{
Incorporación de aditivos en hormigones reciclados para modificar sus propiedades
}

Juan Manuel Moro ${ }^{1}$, Romina Soledad Meneses ${ }^{1}$, Lilian Señas ${ }^{1}$, Carla Priano ${ }^{1}$, Néstor Francisco Ortega ${ }^{1}$ y Raquel René Aveldaño ${ }^{1}$

\section{Resumen}

Para realizar construcciones más sustentables, se utilizan hormigones donde un porcentaje del agregado grueso se reemplaza por hormigón triturado proveniente de la demolición de estructuras cuya vida útil ha finalizado. Por ello, la evaluación de las propiedades de estos hormigones resulta importante. En este trabajo, se estudiaron propiedades mecánicas (resistencia a compresión y tracción indirecta) y físicas relacionadas con la estructura de poros (absorción capilar) de hormigones elaborados con agregado grueso reciclado. Para ello, se moldearon probetas de hormigón con distinta relación agua/cemento y diferentes aditivos: Incorporador de Aire, Superfluidificante e Incorporador de Aire junto con Superfluidificante. Se analizaron las propiedades antes mencionadas para determinar las posibilidades y limitaciones de aplicación de las distintas mezclas ensayadas.

Palabras clave: hormigón reciclado, residuos de construcción, aditivos, construcción sustentable.

1 Departamento de Ingeniería, Universidad Nacional del Sur, Argentina. 


\begin{abstract}
In order to build more sustainable structures, it is used crushed concrete from demolished buildings (with no useful life) as part of the coarse aggregate. For this reason, evaluating these concrete properties is important. They were studied mechanical (compressive and indirect tensile strength) and physical properties related to the pore structure (capillary absorption) of concretes made with recycled coarse aggregate. For this purpose, they were molded concrete samples using different water/cement ratios and different additives such as, air-entraining, superplasticizer and air-entraining plus superplasticizer. The above-mentioned properties were analyzed, to be able to determine the potential and limitations of application of these mixtures.
\end{abstract}

Key words: recycled concrete, construction waste, additives, sustainable construction. 


\section{Introducción}

Uno de los materiales más empleado en la industria de la construcción con fines estructurales, es el hormigón armado.

Al finalizar la Segunda Guerra Mundial comenzó la utilización de agregados reciclados para la elaboración de hormigón. La gran cantidad escombros de demolición generados durante la misma, dio lugar a su reutilización principalmente como material de relleno. En particular, en los últimos tiempos, el hormigón de demolición, es uno de los materiales cuya reutilización ha tenido gran desarrollo en muchos países del mundo. El reciclado de materiales de construcción y demolición en la realización de nuevas obras, no sólo es una forma de conservar las materias primas, sino también de reducir el consumo de energía y por lo tanto disminuir los costos de construcción (Servas V., 1981). Muchos investigadores han aplicado con éxito los agregados reciclados de hormigón en (Lin Y. H et al., 2004; Sagoe-Crentsil K.K. et al., 2001; Poon C.S. et al., 2003) o, estructuras subterráneas, fundaciones, y rellenos (Yoda K. et al., 1988).

El reciclado de hormigón ha tenido, en los últimos años, cada vez más aceptación para su utilización en estructuras resistentes. Por este motivo, se han llevado a cabo numerosas investigaciones sobre su utilización, ya sea como agregado fino o grueso, para la elaboración de un nuevo hormigón (Señas L., et al., 2006; Etxeberria M. et al., 2007; Tam W.Y. et al., 2005; Zega C.J. et al., 2010).

El estudio del agregado reciclado de hormigón, presenta dificultades, pues se trata de un material con propiedades heterogéneas, las cuales dependen, del mortero adherido al agregado grueso del viejo hormigón y de su procedencia. Esto se debe, en el primer caso, al grado de trituración y del manipuleo previo a su utilización; y en el segundo, al tipo y destino de estructura, calidad de hormigón, elaboración, curado, etc. Estas variables, hacen que la calidad del agregado reciclado a utilizar sea muy aleatoria, lo cual es necesario tener en cuenta a la hora de realizar algún análisis.

Estos puntos débiles de los agregados reciclados de hormigón, su alta porosidad, elevada cantidad de grietas, posible contenido de cloruros y sulfatos, y otras impurezas y la gran cantidad de mortero de cemento adherido, afectará el rendimiento mecánico del hormigón reciclado (Ryu J.S. 2003). El objetivo para que el hormigón reciclado pueda ser aplicado en hormigones de alta calidad es conocer estas limitaciones.

En este trabajo se presentan distintas variantes para la elaboración de hormigón reciclado y se analizan las posibilidades de aplicación de las mismas. Para ello se moldearon probetas de hormigón, reemplazando parte de su agregado grueso, por agregado grueso reciclado, y se evaluaron propiedades mecánicas (resistencia a compresión y tracción indirecta) y físicas relacionadas con la estructura de 
poros (absorción capilar). Se emplearon distintas relaciones agua/cemento, con la incorporación de dos tipos de aditivos: incorporador de aire y superfluidificante.

\section{Materiales y mezclas}

Se dosificaron hormigones con distintas relaciones agua/cemento: 0,$45 ; 0,50 \mathrm{y}$ 0,60. Para cada una de ellas, se moldearon probetas sin aditivo, denominadas Patrón (T) y con distintos aditivos como: incorporador de aire (I), superfluidificante (F) e incorporador de aire + superfluidificante $(\mathrm{F}+\mathrm{I})$.

En todas las mezclas de hormigón se reemplazó el 75\% del agregado natural por agregado reciclado, por este motivo, se los identifica como HR75.

Cemento: Se usó cemento pórtland normal (CPN (40)) proveniente de una fábrica ubicada en la provincia de Buenos Aires.

Agregado Fino: constituido por arena natural silícea proveniente de un yacimiento ubicado al sur de la Provincia de Buenos Aires, que cumple con las especificaciones de calidad dadas por la norma IRAM 1627 (IRAM 1627,1997).

Tabla 1: Granulometría del agregado fino

\begin{tabular}{|c|c|c|c|c|c|c|c|}
\hline Tamiz & $9.5 \mathrm{~mm}$ & $\mathrm{~N}^{\circ} 4$ & $\mathrm{~N}^{\circ} 8$ & $\mathrm{~N}^{\circ} 16$ & $\mathrm{~N}^{\circ} 30$ & $\mathrm{~N}^{\circ} 50$ & $\mathrm{~N}^{\circ} 100$ \\
\hline $\begin{array}{c}\% \\
\text { acumulado }\end{array}$ & -- & 5 & 16 & 32 & 59 & 84 & 100 \\
\hline
\end{tabular}

Agregados gruesos, se emplearon dos tipos:

a) Natural: constituido por canto rodado cuyo tamaño máximo nominal es 25 $\mathrm{mm}$. Este agregado cumple con los límites granulométricos indicados en la norma IRAM 1627 (IRAM 1627,1997). La granulometría se indica en la tabla 2.

Tabla 2: Granulometría del agregado grueso

\begin{tabular}{|c|c|c|c|c|c|c|}
\hline $\begin{array}{c}\text { Tamiz } \\
(\mathrm{mm})\end{array}$ & 38 & 25 & 19 & 12,5 & 9,5 & 4,8 \\
\hline $\begin{array}{c}\% \\
\text { acumulado }\end{array}$ & -- & 22 & 47 & 75 & 89 & 100 \\
\hline
\end{tabular}

b) Reciclado: el hormigón a reciclar se obtuvo de la rotura de probetas de 
hormigón elaboradas con canto rodado. Para obtener las muestras recicladas, se utilizó una trituradora de mandíbula de laboratorio. Se clasificó el agregado resultante, de tal manera de ajustar la curva granulométrica a la indicada en la tabla 2 , es decir, se tiene una distribución y un tamaño máximo nominal semejante al canto rodado natural.

Se evaluaron algunas propiedades del agregado natural (canto rodado) y del agregado reciclado (hormigón triturado) como: densidad relativa, en condición saturada y superficie seca, absorción de agua en 24 horas (IRAM 1562, 1978) y resistencia a la fragmentación por el método "Los Ángeles" (gradación “A” según la granulometría obtenida, (IRAM 1532, 2009)). En la Tabla 3 se detallan las características estudiadas del agregado grueso natural y del reciclado.

Tabla 3: Características de los Agregados

\begin{tabular}{|c|c|c|c|}
\hline Agregado & $\begin{array}{c}\text { Densidad (sss) (kg/ } \\
\text { dm3) }\end{array}$ & Absorción (\%) & $\begin{array}{c}\text { Coeficiente } \\
\text { Los Ángeles } \\
(\%)\end{array}$ \\
\hline C. Rodado & 2,66 & 0,4 & 16 \\
\hline Reciclado & 2,39 & 4,7 & 30 \\
\hline
\end{tabular}

Con el fin de analizar el comportamiento del hormigón reciclado elaborado con la incorporación de distintos aditivos, se realizaron diferentes mezclas, cuyas dosificaciones están detalladas en las tablas siguientes. Los hormigones se proyectaron teniendo en cuenta que cumplieran con un ámbito de consistencia de 8-10 cm, medida con el cono de Abrams (IRAM 1536, 1978). Todas las muestras presentaron un aspecto aceptable, sin indicios de segregación ni exudación. Se midió el porcentaje de aire (IRAM 1602,1988) en todos los hormigones que tenían aditivo incorporador de aire (I y $\mathrm{F}+\mathrm{I})$.

Los hormigones elaborados con agregados gruesos reciclados secos y arena natural demandan, para obtener una misma consistencia, aproximadamente un 5\% más de agua que los hormigones convencionales debido a su mayor capacidad de absorción (Di Maio A. 2004; Hansen T.C. and Narud H.; 1983).

Tabla 4: Proporciones de la serie de mezclas con $\mathrm{a} / \mathrm{c}=0,45$

\begin{tabular}{|c|c|c|c|c|}
\hline Mezcla HR75 & T & F & I & F+l \\
\hline $\begin{array}{c}\text { Cemento } \\
\left(\mathrm{kg} / \mathrm{m}^{3}\right)\end{array}$ & 390 & 367 & 390 & 367 \\
\hline Agua (I) & 175 & 165 & 175 & 165 \\
\hline
\end{tabular}




\begin{tabular}{|c|c|c|c|c|}
\hline $\begin{array}{c}\text { Canto rodado } \\
\left(\mathrm{kg} / \mathrm{m}^{3}\right)\end{array}$ & 263 & 263 & 263 & 263 \\
\hline $\begin{array}{c}\text { Agr. reciclado } \\
\left(\mathrm{kg} / \mathrm{m}^{3}\right)\end{array}$ & 699 & 699 & 699 & 699 \\
\hline $\begin{array}{c}\text { Porcentaje aire } \\
\text { medido (\%) }\end{array}$ & $\ldots-$ & $\ldots-$ & 4,0 & 4,0 \\
\hline
\end{tabular}

Tabla 5: Proporciones de la serie de mezclas con $\mathrm{a} / \mathrm{c}=0,50$

\begin{tabular}{|c|c|c|c|c|}
\hline Mezcla HR75 & T & F & I & F+l \\
\hline $\begin{array}{c}\text { Cemento } \\
\left(\mathrm{kg} / \mathrm{m}^{3}\right)\end{array}$ & 350 & 320 & 350 & 320 \\
\hline Agua (l) & 175 & 160 & 175 & 160 \\
\hline $\begin{array}{c}\text { Canto rodado } \\
\left(\mathrm{kg} / \mathrm{m}^{3}\right)\end{array}$ & 263 & 263 & 263 & 263 \\
\hline $\begin{array}{c}\text { Agr. reciclado } \\
\left(\mathrm{kg} / \mathrm{m}^{3}\right)\end{array}$ & 699 & 699 & 699 & 699 \\
\hline $\begin{array}{c}\text { Porcentaje aire } \\
\text { medido (\%) }\end{array}$ & $-\ldots$ & $-\ldots$ & 4,1 & 4,5 \\
\hline
\end{tabular}

Tabla 6: Proporciones de la serie de mezclas con $\mathrm{a} / \mathrm{c}=0,60$

\begin{tabular}{|c|c|c|c|c|}
\hline Mezcla HR75 & T & F & I & F+l \\
\hline $\begin{array}{c}\text { Cemento } \\
\left(\mathrm{kg} / \mathrm{m}^{3}\right)\end{array}$ & 290 & 275 & 290 & 275 \\
\hline Agua (l) & 175 & 165 & 175 & 165 \\
\hline $\begin{array}{c}\text { Canto rodado } \\
\left(\mathrm{kg} / \mathrm{m}^{3}\right)\end{array}$ & 263 & 263 & 263 & 263 \\
\hline $\begin{array}{c}\text { Agr. reciclado } \\
\left(\mathrm{kg} / \mathrm{m}^{3}\right)\end{array}$ & 699 & 699 & 699 & 699 \\
\hline $\begin{array}{c}\text { Porcentaje aire } \\
\text { medido (\%) }\end{array}$ & $-\ldots$ & --- & 5,0 & 5,5 \\
\hline
\end{tabular}

Se hicieron probetas cilíndricas de 15 × $30 \mathrm{~cm}$ para realizar ensayos físicos de capacidad y velocidad de succión capilar (IRAM 1871, 2004) y mecánicos: resistencia a compresión (IRAM 1546, 1992) y tracción indirecta (IRAM 1658, 1995). Se moldearon y curaron de acuerdo con la normativa vigente en nuestro país (IRAM 1534, 2004). 


\section{Resultados y discusión}

Los resultados obtenidos sobre la caracterización de las propiedades mecánicas del hormigón (resistencia a compresión y tracción indirecta) se detallan en la Tabla 7. Los valores de resistencia tabulados corresponden al promedio de tres determinaciones realizadas con probetas semejantes. Los resultados de las propiedades vinculadas con la estructura de poros (capacidad y velocidad de succión capilar) de las muestras con incorporación de aditivos, se detallan en la Tabla 8.

Tabla 7: Características del hormigón empleado en las probetas ensayadas. Resistencia a compresión y tracción indirecta.

\begin{tabular}{|c|c|c|c|}
\hline HR75 & $\begin{array}{c}\text { Relación } \\
a / c\end{array}$ & Compresión [MPa] & $\begin{array}{c}\text { Tracción Indirecta } \\
{[\mathrm{MPa}]}\end{array}$ \\
\hline $\begin{array}{c}\mathrm{T} \\
\mathrm{I} \\
\mathrm{F} \\
\mathrm{F}+\mathrm{I}\end{array}$ & 0,45 & $\begin{array}{l}36.3 \\
33.6 \\
38.9 \\
36.7\end{array}$ & $\begin{array}{l}3.2 \\
3.6 \\
2.7 \\
3.2\end{array}$ \\
\hline $\begin{array}{c}T \\
\mathrm{I} \\
\mathrm{F} \\
\mathrm{F}+\mathrm{I}\end{array}$ & 0,50 & $\begin{array}{l}34.1 \\
31.5 \\
35.2 \\
34.3\end{array}$ & $\begin{array}{l}2,7 \\
2.9 \\
3.2 \\
3.4\end{array}$ \\
\hline $\begin{array}{c}T \\
\mathrm{I} \\
\mathrm{F} \\
\mathrm{F}+\mathrm{I}\end{array}$ & 0,60 & $\begin{array}{l}33.1 \\
22.2 \\
34.5 \\
33.3\end{array}$ & $\begin{array}{l}2,5 \\
2.4 \\
3.4 \\
2.3\end{array}$ \\
\hline
\end{tabular}

Tabla 8: Capacidad y velocidad de succión capilar.

\begin{tabular}{|c|c|c|c|c|c|}
\hline HR75 & $\begin{array}{c}\text { Relación } \\
\text { a/c }\end{array}$ & $\begin{array}{c}\text { Capacidad } \\
\text { de Succión } \\
\text { Capilar }\left[\mathrm{g} / \mathrm{m}^{2}\right]\end{array}$ & $\begin{array}{l}\text { [\% respecto } \\
\text { al patrón] }\end{array}$ & $\begin{array}{c}\text { Velocidad de } \\
\text { Succión Capilar } \\
{\left[\mathrm{g} / \mathrm{m}^{2} \mathrm{~s}^{1 / 2}\right]}\end{array}$ & $\begin{array}{l}\text { [\% respecto } \\
\text { al patrón] }\end{array}$ \\
\hline $\begin{array}{c}T \\
\mathrm{I} \\
\mathrm{F} \\
\mathrm{F}+\mathrm{I}\end{array}$ & 0,45 & $\begin{array}{l}7009 \\
6782 \\
6839 \\
6494\end{array}$ & $\begin{array}{c}100 \\
97 \\
98 \\
93\end{array}$ & $\begin{array}{c}11,8 \\
9,8 \\
11,3 \\
10,3\end{array}$ & $\begin{array}{c}100 \\
83 \\
96 \\
87\end{array}$ \\
\hline $\begin{array}{c}T \\
I \\
F \\
F+I\end{array}$ & 0,50 & $\begin{array}{l}7069 \\
5517 \\
5862 \\
5115\end{array}$ & $\begin{array}{c}100 \\
78 \\
83 \\
72\end{array}$ & $\begin{array}{c}12,0 \\
10,4 \\
10,0 \\
7,8\end{array}$ & $\begin{array}{c}100 \\
87 \\
83 \\
65\end{array}$ \\
\hline $\begin{array}{c}T \\
\mathrm{I} \\
\mathrm{F} \\
\mathrm{F}+\mathrm{I}\end{array}$ & 0,60 & $\begin{array}{l}8046 \\
6667 \\
6724 \\
5920\end{array}$ & $\begin{array}{c}100 \\
83 \\
84 \\
74 \\
\end{array}$ & $\begin{array}{c}17,3 \\
11,6 \\
11,0 \\
9,5\end{array}$ & $\begin{array}{c}100 \\
67 \\
64 \\
55 \\
\end{array}$ \\
\hline
\end{tabular}


La resistencia a la fragmentación por el método "Los Ángeles" obtenido para los agregados reciclados fue aproximadamente el doble, respecto al de los agregados naturales. Aún así, ambos resultados de pérdida se mantienen por debajo del límite indicado como máximo en el Reglamento CIRSOC-201, 6.3.1.2.3 c) CIRSOC 201. Este importante aumento en la pérdida por desgaste se atribuye al elevado porcentaje de mortero y/o pasta adherido al agregado natural, como así también a la presencia de partículas compuestas únicamente de mortero.

La densidad del agregado reciclado resultó ser un $10 \%$ menor, respecto a la del canto rodado, mientras que la absorción fue muy superior a la del agregado natural. Ambas diferencias se deben a que el hormigón triturado tiene un porcentaje de mortero adherido, de menor densidad y con mayor absorción que el canto rodado original, e inclusive puede estar constituido por mortero únicamente.

En las Tablas 7 y 8 se aprecia que los hormigones elaborados con menor relación agua/cemento tienen mayor resistencia y menor absorción.

En todas las mezclas, tanto la capacidad como la velocidad de succión capilar, son elevadas respecto a las de hormigones elaborados en forma convencional. Esto se debe al hecho de que los agregados de hormigón triturado poseen una mayor porosidad.

Para todas las relaciones agua/cemento estudiadas, las muestras con incorporación de aditivos presentaron una disminución en los valores de capacidad y velocidad de succión capilar respecto a las muestras patrón, siendo la más efectiva la combinación de superfluidificante más incorporador de aire $(\mathrm{F}+\mathrm{I})$. Esta disminución fue más significativa en los hormigones de mayor relación a/c.

El uso de incorporador de aire, resultó más beneficioso desde el punto de vista de la absorción capilar en los hormigones de relación agua/cemento de 0,60 , pero generó una importante disminución de la resistencia a compresión (33\%). Para los hormigones con relación agua/cemento de 0,45 , la disminución de la absorción capilar y de la resistencia a compresión fue de un 3\% y un 7\%, respectivamente, y para aquellos con relación agua/cemento de 0,50 , la disminución de la absorción capilar y de la resistencia a compresión fueron de un $22 \%$ y un $8 \%$, respectivamente.

Por su parte, los hormigones con la incorporación del superfluidificante, además de mostrar mejor aspecto y no incorporar aire, tuvieron un leve aumento de la resistencia mecánica. Este aumento fue de un 7\%, para los hormigones elaborados con una relación agua/cemento de 0,45 , de un $3 \%$ para aquellos con relación agua/ cemento de 0,50 y de un $4 \%$, para los hormigones con mayor relación agua/cemento.

La incorporación simultánea de estos dos aditivos, además de disminuir la absorción capilar, hizo que la resistencia mecánica sea, en términos generales, similar al hormigón patrón, al que no se le incorporaron aditivos. 


\section{Conclusiones}

Con respecto a los ensayos realizados sobre el agregado reciclado, estos mostraron que, en comparación con el canto rodado, la densidad fue un $10 \%$ menor, la absorción de agua experimentó un importante aumento y la resistencia a la fragmentación por el método "Los Ángeles" aumentó, aproximadamente, en un $100 \%$.

De acuerdo a los resultados experimentales se demostró que es factible obtener un material de buena prestación, como para ser utilizado en estructuras resistentes, con el reemplazo de un alto porcentaje de agregado natural por agregado reciclado. Los valores de absorción capilar son elevados respecto a los hormigones elaborados en forma convencional, esto se debe al hecho de que los agregados de hormigón triturado poseen una mayor porosidad. Por lo tanto, el hormigón con agregado reciclado es un material más vulnerable frente al ingreso de agentes externos que pueden producir reacciones deletéreas y afectar su durabilidad.

El uso de aditivos es una alternativa para mejorar la performance del hormigón reciclado. Pero es importante seguir investigando en esta línea de trabajo, y poder determinar, si este tipo de hormigones pueden llegar a tener las mismas o muy similares características que aquellos elaborados sin el uso de agregados reciclados.

\section{Agradecimientos}

Los autores desean expresar su agradecimiento al personal del Laboratorio de Modelos Estructurales y del Laboratorio de Estudio y Ensayos de Materiales, U.N.S., por la amplia colaboración prestada para la elaboración de este trabajo. Además, se agradece a las Secretarías General de Ciencia y Tecnología de la Universidad Nacional del Sur y al Departamento de Ingeniería por su aporte económico e institucional necesarios para la materialización de estas investigaciones. 


\section{Referencias}

CIRSOC, Reglamento 201 (1984): Proyecto, Cálculo y Ejecución de Estructuras de Hormigón Armado y Pretensado. Tomo 1, Cap. 6, Buenos Aires.

Di Maio A. (2004). Reciclado de hormigones, Nuevos hormigones y sus aplicaciones, Jornadas organizadas por el LEMIT-CIC, La Plata.

Etxeberria M., Vázquez E., Marí A. and Barra M. (2007). Influence of amount of recycled coarse aggregates and production process on properties of recycled aggregate concrete, Cement and Concrete Research, Vol. 37, 735-742.

Hansen T.C. and Narud H. (1983). Strength of recycled concrete made from crushed concrete coarse aggregate, Concrete International, January, 79-83.

Instituto Argentino de Racionalización de Materiales (IRAM) 1562 (1978): Hormigón fresco de cemento pórtland. Método de determinación de la densidad, el rendimiento y el contenido de agua, Buenos Aires.

Instituto Argentino de Racionalización de Materiales (IRAM) 1532 (2009): Agregados. Determinación de la resistencia a la fragmentación por el método "Los Ángeles", Buenos Aires.

Instituto Argentino de Racionalización de Materiales (IRAM) 1536 (1978): Hormigón fresco de cemento pórtland. Método de ensayo de la consistencia utilizando el tronco de cono, Buenos Aires.

Instituto Argentino de Racionalización de Materiales (IRAM) 1602 (1988): Determinación del contenido de aire incorporado al hormigón fresco, Buenos Aires.

Instituto Argentino de Racionalización de Materiales (IRAM) 1871 (2004). Método de ensayo para determinar la capacidad y la velocidad de succión capilar de agua del hormigón endurecido, Buenos Aires.

Instituto Argentino de Racionalización de Materiales (IRAM) 1546 (1992). Hormigón de cemento portland. Método de ensayo de compresión, Buenos Aires.

Instituto Argentino de Racionalización de Materiales (IRAM) 1658 (1995). Hormigón. Método de ensayo de tracción simple por compresión diametral, Buenos Aires. 
Instituto Argentino de Racionalización de Materiales (IRAM) 1534 (2004). Hormigón. Preparación y curado de probetas en laboratorio para ensayos de compresión y tracción por compresión diametral, Buenos Aires.

Instituto Argentino de Racionalización de Materiales (IRAM) 1627 (1997): Agregados. Granulometría de los agregados para hormigón, Buenos Aires.

Lin Y.H., Tyan Y.Y., Chang T.P. and Chang C.Y. (2004). An assessment of optimal mixture for concrete made with recycled concrete aggregates. Cement and Concrete Research 34, 1373-1380.

Poon C.S., Azhar S. and Kou S.C. (2003), Recycled aggregates for concrete applications, Materials Science and Technology in Engineering Conference, Now, New and Next.

Ryu J.S. (2003). An experimental study on the effect of recycled aggregate on concrete properties, Magazine of Concrete Research, 54, 1, 7-12.

Sagoe-Crentsil K.K., Brown T. and Taylor A.H. (2001). Performance of concrete made with commercially produced coarse recycled concrete aggregate. Cement and Concrete Research 31, 707-712.

Señas L., Priano C., Valea J. and Cabo G. (2006). Reciclado de Hormigones de Canto Rodado Patagónico; $16^{\circ}$ Reunión Técnica de la Asociación Argentina de Tecnología del Hormigón, Mendoza

Servas V. (1981). Recycling concrete materials, Civil Eng., 9, 45-53.

Tam W.Y., Vivian, Gao X.F. and Tam C.M. (2005). Microstructural analysis of recycled aggregate concrete produced from two-stage mixing approach, Cement and Concrete Research, Vol. 35, 1195- 1203.

Yoda K., Yoshikane T., Nakashima Y. and Soshiroda T. (1988). Recycled cement and recycled concrete in Japan, Demolition and Reuse of Concrete and Masonry: Reuse of Demolition Waste, Chapman and Hall, London, 527- 536.

Zega C.J., Villagrán Zaccardi Y.A. and Di Maio A.A. (2010). Effect of natural coarse aggregate type on the physical and mechanical properties of recycled coarse aggregates. Materials and Structures, Vol. 43, 1-2. 
